\title{
Eavesdropping on the two-way quantum communication protocols with invisible photons
}

\author{
Qing-yu Cai \\ State Key Laboratory of Magnetics Resonance and Atomic and Molecular Physics, \\ Wuhan Institution of Physics and Mathematics, \\ the Chinese Academy of Sciences, Wuhan 430071, China
}

\begin{abstract}
The crucial issue of quantum communication protocol is its security. In this paper, we show that all the deterministic and direct two-way quantum communication protocols, sometimes called pingpong (PP) protocols, are insecure when an eavesdropper uses the invisible photon to eavesdrop on the communication. With our invisible photon eavesdropping (IPE) scheme, the eavesdropper can obtain full information of the communication with zero risk of being detected. We show that this IPE scheme can be implemented experimentally with current technology. Finally, a possible improvement of PP communication protocols security is proposed.
\end{abstract}




\section{INTRODUCTION}

Quantum communication, or more precisely called quantum key distribution (QKD), is a physically secure method for the distribution of a secure key between to distant partners, Alice and Bob, who share a quantum channel and public authentificated channel [1]. Since the pioneer work of QKD by Bennett and Brassward in 1984 (BB84) [2], many theoretical proposals [3] and experimental realizations [4] have been presented. With the efforts of physicists and engineers, QKD may be the first commercial application of quantum physics at single photon level [1].

The crucial issue of a QKD protocol is its security. In [5], Brassard, Lütkenhaus, Mor and Sanders presented a criteria for a protocol which can be considered as practical and secure: In order to be practical and secure, a quantum key distribution scheme must be based on existing - or nearly existing - technology, but its security must be guaranteed against an eavesdropper with unlimited computing power whose technology is limited only by the laws of quantum mechanics. Along this line, we can present an eavesdropping scheme on the deterministic and direct two-way quantum communication protocols to gain full information of the key without being detected. The first deterministic and direct two-way quantum communication protocol, sometimes called ping-pong (PP) protocol, was presented by Boström and Felbinger [6] and its security was extensively studied [7]. After that this PP protocol was enhanced with dense coding feature [8,9]. And then it was extended to single photon implementation [10-12].

In this paper, an invisible photon eavesdropping (IPE) scheme is presented, which reveals that the PP-type protocols are insecure under the eavesdropper-Eve's invisible photon eavesdropping attack. In the IPE scheme, Eve first selects a photon which is insensitive for Alice and Bob's single photon detectors, i.e., Eve's such photon is invisible for Alice and Bob. She adds this invisible photon to the travel pulse and forwards them to Alice. After Alice's encoding operation, Eve captures her invisible photon and performs a measurement to draw Alice's encoding message. Because Alice's single photon detector is insensitive to Eve's invisible photon, Alice and Bob can not detect Eve's eavesdropping attack when they check the fidelity of their photons (or the entangled photon pairs). In this way, Eve can gain full information of the communication without being detected. We show the experimental possibility of this IPE scheme. Finally, we present a possible improvement of the PP-type 
protocols.

\section{EAVESDROPPING ON THE PP-TYPE PROTOCOLS}

Let us start with the brief description of the PP protocol of Boström and Felbinger [6]. Bob prepares two photons in an entangled state $\left|\Psi^{+}\right\rangle=(|0\rangle|1\rangle+|1\rangle|0\rangle) / \sqrt{2}$ of the polarization degree freedom. He keeps one photon (home photon) in his laboratory, and sends the other photon (travel photon) to Alice through quantum channel. After receiving the travel photon, Alice randomly switches the control mode and message mode. In control mode, Alice measures the polarization photon and announces the measurement result in the classical public channel. Bob also switches to control mode and measures the home photon in the same basis after he received Alice's announcements. In the absence of Eve, both results should be anticorrelated. In the message mode, Alice performs a unitary operation $Z^{j}$ to encode her message $j \in\{0,1\}$ on the travel photon, where $Z=|0\rangle\langle 0|-| 1\rangle\langle 1|$. Then Alice sends the travel photon to Bob. Bob performs a decoding measurement to draw the information Alice encoded. When the measurement result is $\left|\Psi^{+}\right\rangle$, Bob knows that $j=0$. Likewise, Bob's measurement result $\left|\Psi^{-}\right\rangle$denotes $j=1$. In order to detect Eve's eavesdropping hidden in the channel losses and Eve's attack without eavesdropping [7], Alice and Bob may publish some of their bits to estimate the quantum bit error rate (QBER) at the end of the key distribution. If both the fidelity of the EPR pairs and the QBER are normal, Alice and Bob will use their encoding bits to generate the raw key. After the error-correcting and privacy amplification, Alice and Bob can get the final key.

However, such final key is not secure because of the potential IPE attack. Eve's IPE scheme can be described as follow: Eve first prepares a photon in the states $(|0\rangle+|1\rangle) / \sqrt{2}$. She adds this photon to Bob's travel photon pulse and forwards them to Alice in line B to A. Since the number of photons and the polarization of photon are communicative, Eve's additional photon does not disturb the state of the travel photon. In message mode, Alice sends the travel photon back to Bob after her encoding operation. In line A to B, Eve captures her photon and performs a measurement to draw Alice's encoding message. Eve's measurement result $(|0\rangle+|1\rangle) / \sqrt{2}$ denotes that Alice's encoded message is $j=0$, and $(|0\rangle-|1\rangle) / \sqrt{2}$ denotes $j=1$. Sometimes, after receiving the travel qubit, Alice may switch to control mode. In control mode, Alice measures the travel photon in the polarization basis. 
Since Alice's single photon detector is insensitive to Eve's spy photon, Alice can not find out Eve's photon in control mode. In this way, Eve can obtain full of Alice's information with zero risk.

Likewise, Eve can use her IPE scheme in the single photon PP protocol [10-12]. In a single photon PP protocol, Bob first prepares a polarization photon and then sends it to Alice. After receiving the photon, Alice may switch between control mode and message mode randomly. In message mode, she encodes her message on the photon and then sends it back to Bob. Bob performs a measurement on the photon to gain Alice's encoding message. In control mode, Alice measures the photon and publishes her measurement results. Bob also switches to control mode and publishes the state of the photon to estimate the fidelity of the travel photons. At the end of the key distribution, Alice and Bob publish some of their encoding and decoding message to estimate the QBER. In order to gain Alice's encoding information, Eve can add an invisible photon to the travel photon in line B to A and captures her photon in line A to B. She measures her photon to gain Alice's encoding message precisely. In this way, Eve can also obtain full of Alice's information without being detected.

In the case of dense coding protocols [8,9], Eve can also obtain full of Alice's information with zero risk. In the dense coding protocol, one travel photon can be encoded with two-bit information. On this occasion, Eve can prepare an entangled photon pair first. She stores one photon and sends the other one to Alice to gain information. Also, photons Eve uses are invisible for Alice.

\section{EXPERIMENTAL POSSIBILITY OF THE IPE SCHEME.}

In experiment, the single photon detector is only sensitive to a special wavelength, and it can be used near such wavelength. E.g., a commercial silicon-based single photon detector is used for near infrared wavelengths $600 \mathrm{~nm}$ to $900 \mathrm{~nm}$. And a single-electron transistor consisting of a semiconductor quantum dot in high magnetic field can help one to detect the single far-infrared photons in the wavelength range 175-210 microm [13]. Thus, Eve can select a wavelength which is far away from the wavelength Alice and Bob use. In control mode, Alice can not detect Eve's invisible photon. In message mode, Alice only performs encoding operations which can be implemented by using some linear optical elements. Be- 
cause the number of photons and the polarization of the photon are communicative, Eve's eavesdropping action does not disturb the state of Alice and Bob's travel photon. Eve can distinguish her photon from the travel photon because of the different wavelengths, which can be realized with a spectroscope experimentally. In this case, Eve can gain full of Alice's information with zero risk of being detected. In fact, Eve does not need to add her invisible to Bob's photon pulse. She can send her invisible photon freely in line B to A and measure her photon in line A to B after the spectroscope to gain Alice's encoding information. This can be implemented experimentally with current technology.

\section{POSSIBLE MODIFICATION OF THE PP-TYPE PROTOCOLS WITH FIL- TER}

Essentially, such IPE attack is trojan horse attack [14] which aims the special property of the PP-type quantum communication protocols. The favor of the PP protocols is that information can be transmitted in a deterministic and direct way. In order to defeat Eve's IPE attack, the PP-type protocols should be modified. A possible modification is proposed as below.

Alice adds a filter in her laboratory first [15]. All Bob's photon pulses should pass through her filter first. Only wavelengths close to the operating wavelength can be let in. Thus, Eve's invisible photons can be filtered out by using the filter. If Eve's spy photons can not be filtered out, they will be detected in Alice's photon detection apparatus. These days, the bandwidth of optical devices is as narrows as 0.1 to $0.01 \mathrm{~nm}$ which is comparable to the laser linewidth. An optical grating to filter out unwanted frequencies may be used in combination with such the narrow bandwidth devices [15]. In this way, Alice and Bob can defeat Eve's IPE attack.

\section{REMARK.}

In [16], Kye, Kim, Kim, and Park (KKKP) have present a QKD protocol with blind polarization bases. (a.1) Alice chooses a random value of angle $\theta$ and prepares a photon state with polarization of that angle. She then sends the qubit to Bob. (a.2) Bob also chooses another random value of angle $\phi$ and further rotates the polarization direction of 
the received state by $\phi$ and then returns to Alice. (a.3) Alice rotates the polarization angle by $-\theta$, and then encodes the message by rotating the polarization angle $\pm \frac{\pi}{4}$. And then she sends the photon to Bob. (a.4) After rotating the polarization angle by $-\phi$, Bob measures the photon to draw Alice's encoding message. In this way, information can be transmitted directly with blind bases. In fact, KKKP's protocol is not a two-way ping-pong protocol but a 3-way protocol, i.e., a key has to travel 3 times between Alice and Bob. Also, in KKKP's protocol, Eve can first send an n-photon pulse before Alice's encoding operations, and then performs measurement on her $n$ photons after receiving her $n$ photons from Alice. Alice can estimate the state of the $\mathrm{n}$ photons precisely by increasing $\mathrm{n}$. However, if Alice's rotating angle $\theta$ is random, Eve can not distinguish which encoding operation Alice performed. In KKKP, a simple IPE scheme is useless because of the blind bases. Even if Eve uses the sophisticated photon-number-splitting attack scheme $[17,15]$, she can only get benefits from the imperfect single-photon sources.

\section{SUMMARY}

In summary, we have presented an IPE scheme on the PP type quantum communication protocols. Using our IPE scheme, an eavesdropper can gain full information of the communication with zero risk. We have shown the experimental possibility of the IPE scheme. We also suggest the possible improvements of the PP type protocols to fulfill the condition of the security against the IPE attack.

\section{ACKNOWLEDGEMENT}

I am grateful to M. S. Kim for the detailed discussions on this work, especially the idea of filtering the invisible photons. This work is supported by the Nature Science Foundation of China under Grant No. 10447140 and 10504039.

\section{REFERENCES}

[1] N. Gisin, G. Ribordy, W. Tittel, and H. Zbinden, Rev. Mod. Phys. 74, 145 (2002). 
[2] C. H. Bennett and G. Brassward, In Procedings IEEE International Conference on Computers, Systems and Signal Processing, Bangalore, India (IEEE, New York, 1984), 99. 175-179.

[3] A. K. Ekert, Phys. Rev. Lett. 67, 661 (1991); M. Curty, M. Lewenstein, and N. Lükenhaus, Phys. Rev. Lett. 92, 217903 (2004); H.-K. Lo and H. F. Chau, Science 283, 2050 (1999); D. Mayer, J. Assoc. Comput. Mach. 48, 351 (2001); P. W. Shor and J. Preskill, Phys. Rev. Lett. 85, 441 (2000).

[4] C. H. Bennett, F. Bessette, G. Brassward, L. Salvail, and J. Smolin, J. Cryptography 5, 3-28 (1992); For a review, see Ref. [1].

[5] G. Brassward, N. Lükenhaus, T. Mor, and B. C. Sander, Phys. Rev. Lett. 85, 1330 (2000).

[6] K. Boström and T. Felbinger, Phys. Rev. Lett. 89, 187902 (2002).

[7] A. Wójcik, Phys. Rev. Lett. 90, 157901 (2003); Q.-Y. Cai, Phys. Rev. Lett. 91, 109801 (2003).

[8] Q.-Y. Cai and B.-W. Li, Phys. Rev. A 69, 054301 (2004); Nguyen Ba An, Phys. Lett. A 328,6 (2004).

[9] I. P. Degiovanni et al., Phys. Rev. A 69, 032310 (2004); A. Wójcik, Phys. Rev. A 71, 016301 (2005).

[10] Q.-Y. Cai and B.-W. Li, Chin. Phys. Lett. 21, 601 (2004); H. Lü, X.-D. Yan and X.-Z. Zhang, Chin. Phys. Lett. 21, 2340-2342 (2004).

[11] M. Lucamarini and S. Mancini, Phys. Rev. Lett. 94, 140501 (2005).

[12] F.-G. Deng and G. L. Long, Phys. Rev. A 69, 052319 (2004); Holger Hoffmann, K. Boström and T. Felbinger, Phys. Rev. A 72, 016301 (2005); F.-G. Deng and G. L. Long, Phys. Rev. A 70, 012311 (2004).

[13] S. Komiyama, O. Astafiev, V. V. Antonov, T. Kutsuwa, and H. Hirai, Nature 403, 405 (2000).

[14] N. Gisin, S. Fasel, B. Kraus, H. Zbinden and G. Ribordy, quant-ph/0507063.

[15] W.-K. Kye and M. S. Kim, quant-ph/0508028; Private communication with M. S. Kim.

[16] W.-K. Kye, C.-M. Kim, M. S. Kim, and Y.-J. Park, Phys. Rev. Lett. 95, 040501 (2005).

[17] Q.-Y. Cai, Eavesdropping on the "quantum key distribution with blind polarization 
bases" using sophisticated photon-number-splitting attack, (submitted for publication.).

IX. 